\title{
Time to Disease-modifying Antirheumatic Drug Treatment in Rheumatoid Arthritis and Its Predictors: A National, Multicenter, Retrospective Cohort
}

\author{
RUBEN TAVARES, JANET E. POPE, JEAN-LUC TREMBLAY, CARTER THORNE, VIVIAN P. BYKERK, \\ JURIS LAZOVSKIS, KENNETH L.N. BLOCKA, MARY J. BELL, DIANE LACAILLE, CAROL A. HITCHON, \\ AVRIL A. FITZGERALD, WESLEY K. FIDLER, ARTHUR A.M. BOOKMAN, JAMES M. HENDERSON, \\ DIANNE P. MOSHER, DALTON E. SHOLTER, MAJED KHRAISHI, BOULOS HARAOUI, HONG CHEN, \\ XIUYING LI, ANDREAS LAUPACIS, GILLES BOIRE, GEORGE TOMLINSON, and CLAIRE BOMBARDIER
}

\begin{abstract}
Objective. To determine the proportion of patients with rheumatoid arthritis (RA) under rheumatologic care treated with disease-modifying antirheumatic drugs (DMARD) within 6 months from symptom onset and the components of time to treatment and its predictors.

Methods. A historical inception cohort of 339 patients with RA randomly selected from 18 rheumatology practices was audited. The proportion that initiated DMARD treatment within 6 months from symptom onset was estimated using Kaplan-Meier analysis. Time to each component of the care pathway was estimated. Multivariable modeling was used to determine predictors of early treatment using 12 preselected variables available in the clinical charts. Bootstrapping was used to validate the model.

Results. Within 6 months from symptom onset, $41 \%$ (95\% CI 36\%-46\%) of patients were treated with DMARD. The median time to treatment was 8.4 (interquartile range 3.8-24) months. Events preceding rheumatology referral accounted for $78.1 \%$ of the time to treatment. The most prominent predictor of increased time to treatment was a concomitant musculoskeletal condition, such as osteoarthritis or fibromyalgia. The significance of other variables was less consistent across the models investigated. Included variables accounted for $0.69 \pm 0.03$ of the variability in the model.

Conclusion. Fewer than $50 \%$ of patients with RA are treated with DMARD within 6 months from symptom onset. Time to referral to rheumatology represents the greatest component delay to treatment. Concomitant musculoskeletal condition was the most prominent predictor of delayed initiation of DMARD. Implications of these and other findings warrant further investigation. (First Release Aug 15 2012; J Rheumatol 2012;39:2088-97; doi:10.3899/jrheum.120100)
\end{abstract}

Key Indexing Terms:

RHEUMATOID ARTHRITIS

EPIDEMIOLOGIC FACTORS

DISEASE-MODIFYING ANTIRHEUMATIC DRUGS

PRACTICE PATTERNS

COHORT STUDIES

HEALTH SERVICES RESEARCH

From McMaster University, Hamilton; University of Western Ontario, London; Southlake Regional Health Centre, Newmarket; Mount Sinai Hospital, University of Toronto; Sunnybrook Health Sciences Centre; Toronto Western Hospital; University Health Network; Keenan Research Centre, Li Ka Shing Knowledge Institute of St. Michael's Hospital, University of Toronto, Toronto; St. Joseph's Health Centre, Thunder Bay, Ontario; Centre Hospitalier Régional de Trois-Rivières, Québec City; University of Montreal, Montreal; Université de Sherbrooke, Sherbrooke, Quebec; Dalhousie University, Halifax; Cape Breton Regional Hospital, Sydney, Nova Scotia; University of British Columbia, Vancouver, British Columbia; University of Manitoba, Winnipeg, Manitoba; University of Calgary, Calgary; University of Alberta, Edmonton, Alberta; Fredericton Medical Centre, Fredericton, New Brunswick; and Memorial University, St. John's, Newfoundland, Canada.

Supported by unrestricted educational grants from Amgen Canada Inc./Wyeth Pharmaceuticals, and Schering Canada Inc. R. Tavares received scholarship support from the Canadian Arthritis Network, Edward Dunlop Foundation Ontario Graduate Scholarship in Science and Technology, and the University of Toronto Institute of Medical Science. Dr. Bombardier holds a Pfizer Chair and a Canada Research Chair in Knowledge Transfer for Musculoskeletal Care.
R. Tavares, MBt, MSc, McMaster University; J.E. Pope, MD, MPH, University of Western Ontario; J-L. Tremblay, MD, Centre Hospitalier Régional de Trois-Rivières; C. Thorne, MD, Southlake Regional Health Centre; V.P. Bykerk, MD, Mount Sinai Hospital; M.J. Bell, MD, MSc, Sunnybrook Health Sciences Centre; A.A.M. Bookman, MD, Toronto Western Hospital; H. Chen, MSc; G. Tomlinson, PhD; X. Li, MD, MSc, University Health Network; A. Laupacis, MD, MSc, Keenan Research Centre, Li Ka Shing Knowledge Institute, St. Michael's Hospital; C. Bombardier, MD, University Health Network; J. Lazovskis, MD, Cape Breton Regional Hospital; K.L.N. Blocka, MD; D. Lacaille, MD, MSc, University of British Columbia; C.A. Hitchon, MD, MSc, University of Manitoba; A.A. Fitzgerald, MD, University of Calgary; W.K. Fidler, MD, St. Joseph's Health Centre; J.M. Henderson, MD, Fredericton Medical Centre; D.P. Mosher, MD, Dalhousie University; D.E. Sholter, MD, University of Alberta; M. Khraishi, MB, Memorial University; B. Haraoui, MD, University of Montreal; G. Boire, MD, MSc, Université de Sherbrooke.

Address correspondence to R. Tavares, McMaster University, 501-25 Charlton Avenue East, Hamilton, ON L8N 1Y2, Canada. E-mail: ruben.tavares@sympatico.ca

Accepted for publication June 5, 2012. 
Early, aggressive pharmacological treatment with disease-modifying antirheumatic drugs (DMARD) is recommended as an effective method of controlling synovitis and limiting erosive joint damage in rheumatoid arthritis (RA) ${ }^{1,2,3}$. While randomized controlled trials (RCT) have proven the short-term efficacy of early DMARD interventions ${ }^{4,5,6,7,8}$, the time-dependence of treatment initiation for disease remission and functional outcome has been investigated in cohort studies ${ }^{9,10,11,12}$. Pharmacologic treatment guidelines for RA recommend treatment with DMARD immediately upon the establishment of a diagnosis ${ }^{1,2,3}$.

Establishing a diagnosis of RA near symptom onset is challenging. Many diagnoses remain in the differential and RA symptoms may be underrecognized ${ }^{13}$. The symptoms characterized in classification criteria for $\mathrm{RA}^{14}$ perform well for longstanding or prevalent disease; however, they are less discriminative near symptom onset ${ }^{15,16}$. New criteria designed to classify DMARD-naive patients with a clinical indication for methotrexate therapy may have diagnostic value for early RA ${ }^{17}$. Currently, RA is first established on the clinical grounds of inflammation, morning stiffness, tender and swollen joint counts, and at least 6 to 12 weeks of symptom persistence ${ }^{9,10,13}$.

Time to rheumatologic care and DMARD treatment is increasingly recognized as an important, modifiable aspect of care for RA A $^{18,19,20,21,22,23,24}$. The existing literature varies by geographical location, clinical setting, date, definition of diagnosis, and specific outcome measured. Across these studies, median estimates of time to treatment range from 4 to more than 22 months. Investigations of the component times and predictors of time to DMARD treatment are sparse $^{18,19,20,21,22,24}$.

The 3 objectives of this study were to determine (1) the proportion of Canadian patients with RA under rheumatologic care treated with DMARD within 6 months from symptom onset; (2) the components of the overall time to treatment; and (3) the predictors of time to DMARD treatment in RA.

\section{MATERIALS AND METHODS}

Study design. A Canadian multicenter historical inception cohort of 339 randomly selected patients with RA under rheumatologic care was assembled. Rheumatologist representation from all provinces was sought. On the basis of characteristics available in the Canadian Medical Directory ${ }^{25}$ (sex, decade of graduation, hospital privileges, academic status, and provincial representation), the distribution of these variables was similar between participating and all Canadian rheumatologists ${ }^{26}$. Research ethics board approval was received from each rheumatologist's site.

Patients were included if they had a definitive clinical diagnosis of RA between June 2001 and May 2003 and were 18 years of age or older at the time of diagnosis. In this study, a definitive clinical diagnosis referred to a record in the rheumatologic clinical chart without reference to an alternative diagnosis in the differential. Patients were excluded if they fulfilled any of the following criteria: history of juvenile inflammatory arthritis; change in diagnosis over followup; or a concomitant disease that precluded DMARD treatment.

To eliminate selection bias toward RA patients with shorter time to
DMARD treatment or toward patients under rheumatologic care at the time of the review, randomly selected charts were screened for eligibility. Each participating rheumatologist's administrative billing system was prescreened for patients receiving an International Classification of Diseases, 9th revision, Clinical Modification (ICD-9-CM) diagnosis code of 714, and a first consultation service code (A485A or A135A) between 2001 and 2003 but not necessarily on the same visit (Figure 1). A list of patients satisfying both criteria was randomized and used to screen clinical charts for study eligibility. At each rheumatologist's site, charts were screened until the first of either of the following occurred: 20 eligible charts were identified; or all prescreened charts were reviewed.

A case review form (CRF) was developed from a pilot study of 36 patients from 6 practices ( 6 per practice), selected as described above. An iterative process was used to develop the CRF: suggestions for revisions were collected and implemented after each site's successive chart extraction. A CRF guidance document was created and circulated to all participating sites to promote consistent data extraction in the full study.

Data management and statistical analysis. Sample size. A sample size of 370 was calculated to estimate the proportion of patients with RA treated within 6 months from symptom onset with a 95\% confidence bound of $10 \%$. Using the 10 -events-per-variable convention for multivariable logistic regression modeling ${ }^{27}, 120$ events were required to investigate the effect of 12 independent variables.

Data management. One person manually entered the data collected into a Microsoft Access 2003 database (Microsoft, Redmond, WA, USA). Double data entry was conducted for a random selection of $20 \%$ of charts to estimate the reliability of initial data entry. A double data entry error rate of $<5 \%$ was considered acceptable. Logical and missing data queries were conducted to further increase accuracy of data extraction. Illogical and inconsistent data entries and missing data for variables associated with the primary objective were reconciled with the CRF. Markov chain Monte Carlo multiple imputation ${ }^{28}$ was used to account for missing data among the following variables (percentage of missing data): C-reactive protein

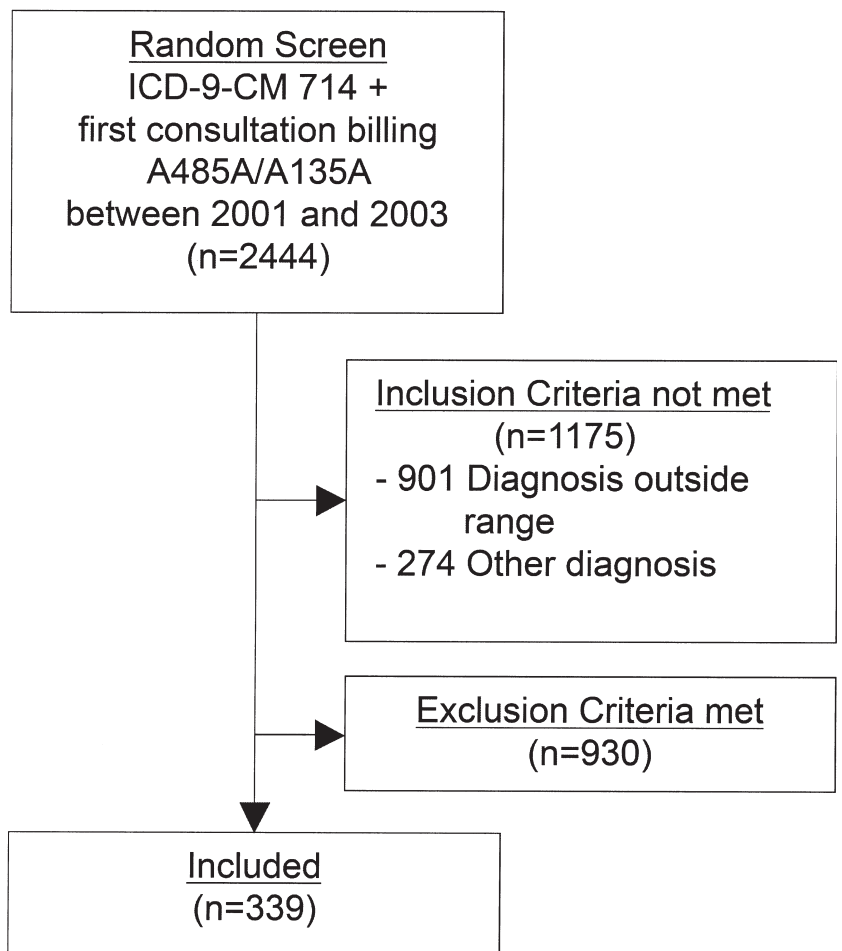

Figure 1. The selection process of patients screened, ineligible, and included in the study. ICD-9-CM: International Classification of Diseases, 9th revision, Clinical Modification. 
(CRP; 56.3\%), tender joint count (32.7\%), swollen joint count (19.8\%), erythrocyte sedimentation rate (ESR; 19.2\%), and rheumatoid factor (RF; $8.6 \%$ ). Five imputations were performed and averaged. Data management queries and statistical analyses were carried out using SAS version 9.2 (SAS, Cary, NC, USA).

Statistical analysis. The proportion of patients treated within 6 months from symptom onset and the median time to treatment were determined. The median time to DMARD treatment was determined using the Kaplan-Meier method. This survival analysis method accounts for the followup time of observations without an event over the followup period by providing censored observations for these times ${ }^{29}$. These censored observations indicate that the followup time was cut off prior to the occurrence of the event. Where symptom onset date and/or DMARD start date were missing in this study, Kaplan-Meier analysis was used to censor these dates. Missing symptom onset $(4.7 \%)$ was censored using the earliest of the following dates: nonsteroidal antiinflammatory drugs (NSAID) start, probable diagnosis, rheumatology referral, first and followup rheumatology visits, diagnosis, and first radiographic evidence of erosive joint damage. Missing DMARD start date $(2.1 \%)$ was censored using the date of chart review. Both the symptom onset date and DMARD start date were missing from the chart of 1 patient $(0.3 \%)$. Complete case estimates of time from symptom onset to DMARD treatment were also reported.

The duration of components of time in the care pathway were estimated from complete data. The duration from symptom onset to each of the following component dates were determined and ordered by increasing magnitude: NSAID start, probable diagnosis, rheumatology referral, first and followup rheumatology visits, confirmed diagnosis, and DMARD start. For patients who consulted multiple rheumatologists, estimates of times from symptom onset to rheumatology referral, and first and followup rheumatology visits, did not represent time to initial rheumatologic care and were therefore excluded.

Two predictive models were tested. A logistic regression model was used to predict a dichotomous outcome: whether or not a patient initiated DMARD treatment within 6 months from symptom onset. A Cox proportional hazards survival analysis was also modeled to predict time to DMARD initiation as a continuous variable, therefore providing a more precise measure of time. The main assumption that hazards are proportional over time was tested ${ }^{30}$. As with the Kaplan-Meier method above, the Cox model made use of the censored data. Model covariables were selected from an informal literature review and in consideration of data available in the clinical charts as determined from pilot testing. The effect size and significance of associations between preselected variables were determined to assess multicollinearity.

Bivariate associations between binary data were determined from OR. Assumptions of normality were tested and bivariate associations of continuous and/or ordinal data across 2 groups were investigated using the Wilcoxon rank-sum test. Bivariate correlations between continuous and/or ordinal data were investigated using Spearman's rank correlation coefficient.

Stepwise predictor selection was used with a multivariable level of significance cutoff of $<50 \%(\mathrm{p}<0.50)$. This stepwise selection threshold was selected as a tradeoff between divergent modeling approaches: those that promote the use of all preselected variables but possibly risk overfitting and stepwise selection techniques that produce more parsimonious models with improved model fit that risk inferior predictive performance ${ }^{27}$. The ability of the model to discriminate between patients at high and low risk of treatment within 6 months from symptom onset was quantified by a measure of concordance, $\mathrm{c}$-index ${ }^{27}$. The logistic regression model was internally validated by determining the percentage covariables selected in a simulation of 200 bootstrap samples ${ }^{31}$.

\section{RESULTS}

Patients. To identify the 339 eligible patients, 2444 charts from the practices of 18 rheumatologists representing 9 provinces and territories were randomly screened (Figure 1). Six rheumatologists who included 106 patients (31.3\%) excluded 801 charts $(86.1 \%)$. To determine if there was selection bias between rheumatologists who excluded the most and fewest charts, the difference in the primary outcome between these 2 groups was tested. The time from symptom onset to DMARD treatment was 239 days [interquartile range (IQR) 109-640] for patients selected by rheumatologists who excluded the majority of the charts compared to 238 (IQR 103-533) days for patients included by the other participating rheumatologists $(\mathrm{p}=0.83)$.

The baseline characteristics for eligible patients were stratified by treatment with DMARD within 6 months from symptom onset (Table 1). Baseline was defined as the period leading up to and including the definitive clinical diagnosis date. The study sample was $75.5 \%$ female and a median of 50 (IQR 41-60) years of age. Baseline disease was characterized by 10 (IQR 6-14) swollen and 14 (IQR 8-20) tender joints, an ESR of 32 (IQR 20-47) $\mathrm{mm} / \mathrm{h}$, and CRP of 28 (IQR 13-38) mg/l. At baseline, 70.5\% $(\mathrm{n}=239)$ were RF-positive. Nearly half the patients $(40.4 \%, \mathrm{n}=137)$ had osteoarthritis, fibromyalgia, osteoporosis, or another concomitant musculoskeletal condition for which DMARD therapy is not indicated, $41.9 \%(\mathrm{n}=142)$ previously consulted another rheumatologist, and $63.1 \%(\mathrm{n}=214)$ were seen by an academic study investigator. The majority $(85.0 \%, \mathrm{n}=288)$ were treated with NSAID prior to the documentation of a definitive diagnosis and $16.2 \%(n=55)$ had radiographic evidence of erosions at baseline.

Time to DMARD treatment. Within the primary endpoint of 6 months, $41 \%$ of patients (95\% CI $36 \%-46 \%, \mathrm{n}=139)$ were treated with DMARD; $21 \%(95 \%$ CI $16 \%-25 \%, \mathrm{n}=$ 70) were treated within 3 months from symptom onset (Figure 2). From Kaplan-Meier analysis, the median time from symptom onset to DMARD treatment was 8.4 (IQR 3.8-24.0) months (range 0-30.6 yrs). Dropping censored data, the time to DMARD treatment was estimated to be 7.7 months (IQR 3.6-18.0), or 233 days (IQR 109-550; range 0-20.7 yrs).

Components of time to DMARD treatment (Figure 3). Relative to symptom onset, the median duration of the components of time to DMARD treatment included the following: 63 days (IQR 0-305) to initiation of NSAID treatment ( $\mathrm{n}=247)$; 181 days (IQR 68-386) to referral to a rheumatologist $(\mathrm{n}=176)$; 182 days (IQR 63-568) to probable RA diagnosis $(\mathrm{n}=230) ; 212$ days (IQR 104-436) to first rheumatology visit $(\mathrm{n}=191)$; and 233 days (IQR 109-550) to initiation of DMARD $(n=317)$. The followup visit with the rheumatologist occurred at 294 days (IQR 157-572; $\mathrm{n}=$ 187), and a definitive RA diagnosis was established at 303 days (IQR 138-803; $\mathrm{n}=323$ ).

Time to NSAID treatment represented $27.0 \%$ of the total time from symptom onset to DMARD treatment. About half $(51.1 \%)$ the total time to DMARD treatment was represent-

Personal non-commercial use only. The Journal of Rheumatology Copyright @ 2012. All rights reserved. 
Table 1. Baseline characteristics* of study participants by time to treatment with DMARD.

\begin{tabular}{lcccc}
\hline & \multicolumn{4}{c}{ Time from Symptom Onset to DMARD Treatment } \\
Variable & Overall, & $<6$ mo, & $\geq 6$ mo, & $\mathrm{p}^{\dagger}$ \\
& $\mathrm{n}=339$ & $\mathrm{n}=139$ & $\mathrm{n}=200$ & \\
\hline Age at symptom onset, yrs, median (IQR) & $50(41-60)$ & $50(40-62)$ & $50(41-59)$ & 0.77 \\
Swollen joint count, median (IQR) & $10(6-14)$ & $11(6-15)$ & $9(5-14)$ & 0.05 \\
Tender joint count, median (IQR) & $14(8-20)$ & $15(9-21)$ & $13(7-19)$ & 0.05 \\
ESR, mm/h, median (IQR) & $32(20-47)$ & $32(20-49)$ & $32(20-46)$ & 0.92 \\
CRP, mg/l, median (IQR) & $28(13-38)$ & $29(16-38)$ & $28(12-38)$ & 0.42 \\
Rheumatoid factor-positive, $\mathrm{n}(\%)$ & $239(70.5)$ & $98(70.5)$ & $141(70.5)$ & 1.00 \\
Male, $\mathrm{n}(\%)$ & $83(24.5)$ & $43(30.9)$ & $40(20.0)$ & 0.02 \\
Concomitant MSK condition, $\mathrm{n}(\%)$ & $137(40.4)$ & $47(33.8)$ & $90(45.0)$ & 0.04 \\
$\quad$ Osteoarthritis & $91(26.8)$ & - & - & - \\
$\quad$ Fibromyalgia & $36(10.7)$ & - & - & - \\
$\quad$ Osteoporosis & $47(13.9)$ & - & - & - \\
$\quad$ Other & $3(0.9)$ & - & - & - \\
Radiographic evidence of erosions, $\mathrm{n}(\%)$ & $55(16.2)$ & $17(12.2)$ & $38(19.0)$ & 0.10 \\
Previously consulted another rheumatologist, $\mathrm{n}(\%)$ & $142(41.9)$ & $67(48.2)$ & $75(37.5)$ & 0.05 \\
Use of NSAID prior to diagnosis, $\mathrm{n}(\%)$ & $288(85.0)$ & $117(84.2)$ & $171(85.5)$ & 0.74 \\
Academic study investigator, $\mathrm{n}(\%)$ & $214(63.1)$ & $98(70.5)$ & $116(58.0)$ & 0.02 \\
\hline
\end{tabular}

* Maximum value recorded in the clinical chart up to the date of diagnosis. ${ }^{\dagger} \mathrm{Z}$ approximation of Wilcoxon rank-sum test for ordinal variables with skewed data distributions; Cochran Mantel-Haenszel chi-square test for $2 \times 2$ tables for binary data, positive vs negative variable. CRP: C-reactive protein; DMARD: disease-modifying antirheumatic drug; ESR: erythrocyte sedimentation rate; IQR: interquartile range; MSK: musculoskeletal; NSAID: nonsteroidal antiinflammatory drug.

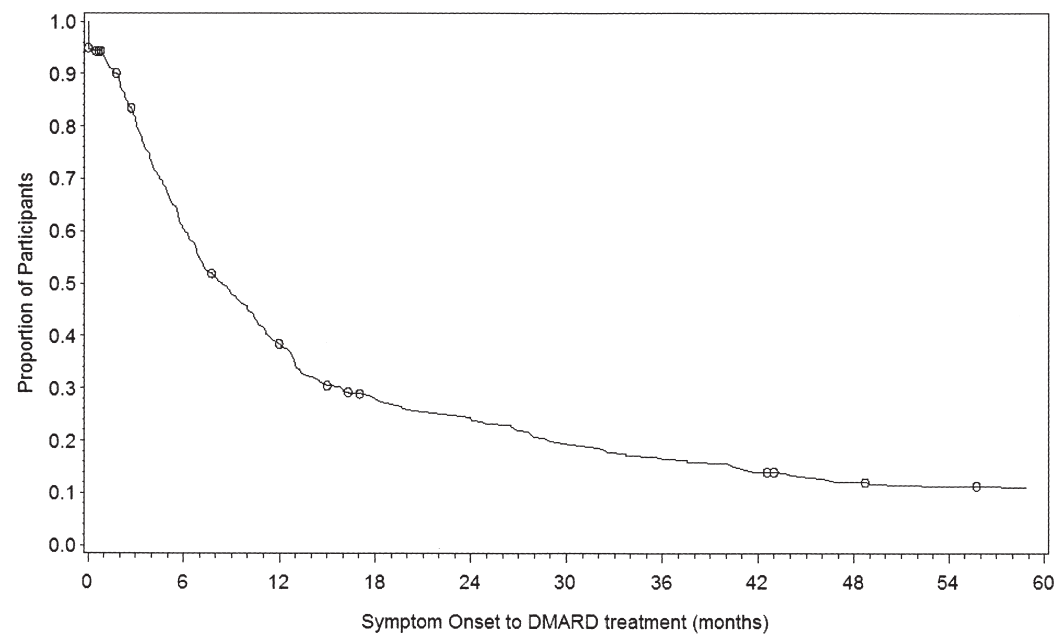

Figure 2. Kaplan-Meier survival analysis of time from symptom onset to initial disease-modifying antirheumatic drug (DMARD) treatment. Results were truncated to the first 60 months. Each symbol represents a censored data point.

ed by the period between the initiation of NSAID treatment and referral to rheumatology. The time of a probable diagnosis corresponded with the time that patients were referred to rheumatologic care. Rheumatology referral wait-time accounted for $12.9 \%$ of the total time to DMARD treatment. The time from first rheumatology visit to DMARD treatment represented $9.0 \%$ of the total time from symptom onset to initiation of DMARD.
For $39.8 \%$ of patients $(\mathrm{n}=135)$, DMARD treatment was initiated in advance of establishment of a definitive diagnosis of RA. Among patients consulting a single rheumatologist, 52.8\% initiated DMARD treatment at their first presentation. The median difference between DMARD initiation and date of first visit was 0 (IQR 0-43) days. The majority of patients who previously consulted another rheumatologist initiated DMARD therapy prior to consulting the study

Personal non-commercial use only. The Journal of Rheumatology Copyright (c) 2012. All rights reserved. 


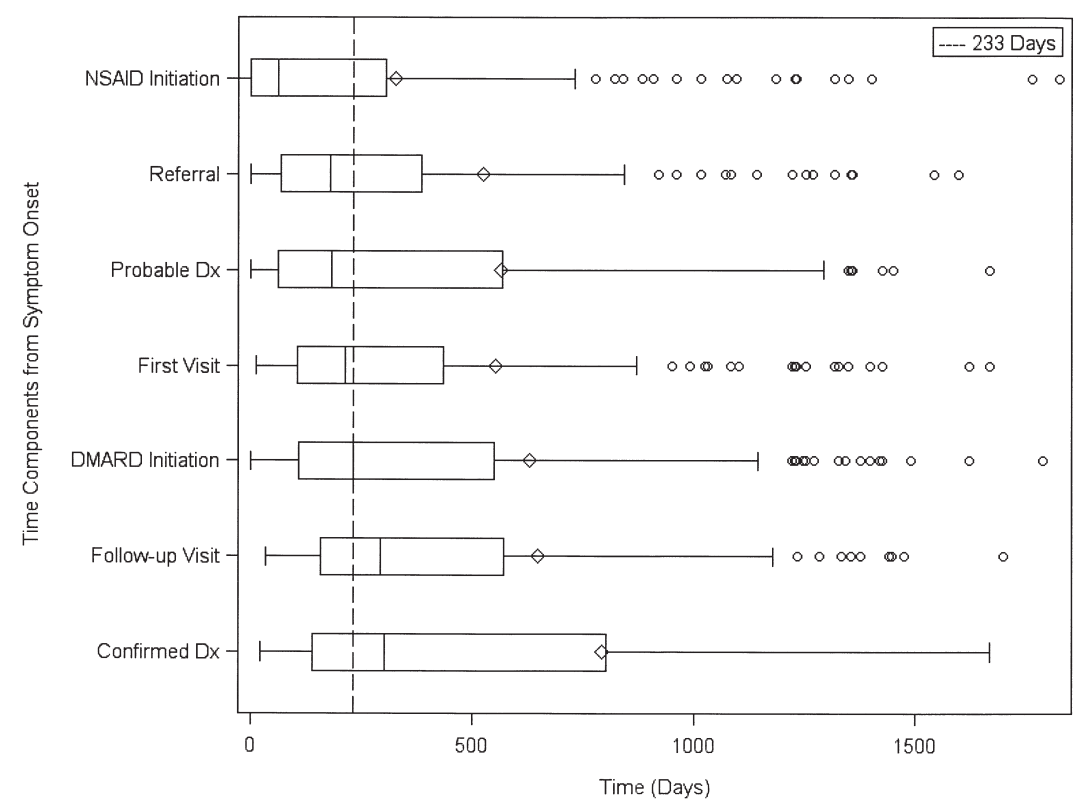

Figure 3. Components of time from symptom onset to disease-modifying antirheumatic drug (DMARD) treatment. Boxes represent 25th-75th percentiles. Lines inside each box represent the median. Each diamond symbol represents the mean. Lines outside each box represent 0 and 100th percentiles. Each circle symbol represents an outlier observation. Vertical broken line denotes median time from symptom onset to DMARD initiation. NSAID: nonsteroidal antiinflammatory drugs.

rheumatologist $(87.4 \%)$. Overall, $68.4 \%$ of patients were either started on DMARD treatment at the first rheumatology visit or were treated by the rheumatologist previously consulted.

Bivariate associations. In bivariate analyses, male sex, absence of a concomitant musculoskeletal condition, previously consulting another rheumatologist, seeing an academic versus a nonacademic study rheumatologist, and higher tender or swollen joint counts were associated with DMARD treatment within 6 months from symptom onset (Tables 1 and 2). Higher tender and swollen joint counts, lack of radiographic evidence of erosions, male sex, absence of a concomitant musculoskeletal condition, previously consulting another rheumatologist, and consulting an academic study investigator were associated with less time from symptom onset to DMARD treatment (Table 3). The effect sizes for significant bivariate associations between covariables were small and did not warrant adjustment for collinearity in multivariate analyses.

Multivariable-adjusted associations. From multivariable logistic regression $\mathrm{p}<0.50$ stepwise selection models (Table 2), absence of a concomitant musculoskeletal condition and previously consulting another rheumatologist were associated with greater odds for treatment within 6 months from symptom onset. The most commonly selected variables across the 200 bootstrap $\mathrm{p}<0.50$ stepwise selection models were previously consulting another rheumatologist $(98.5 \%)$, absence of a concomitant musculoskeletal condition
(96.0\%), consulting an academic investigator (87.5\%), male sex $(84.5 \%)$, and absence of radiographic evidence of erosions at baseline $(81.5 \%)$. The c-index for bootstrap models was $0.69 \pm 0.03$.

In the corresponding Cox proportional hazards model (Table 3$)$, older age at symptom onset $(\mathrm{HR}=1.01, \mathrm{p}=0.02)$ and the absence of a concomitant musculoskeletal condition $(\mathrm{HR}=1.45, \mathrm{p}=0.002)$ were significantly associated with less time to DMARD treatment. The hazard ratios for these 2 variables were constant over time. The association between increasing ESR and time to DMARD treatment (HR $=1.01, \mathrm{p}=0.02)$ was an artifact of using the mean of 5 multiple imputation iterations in the model. When multiple imputation variance was accounted for, this association was not significant.

\section{DISCUSSION}

Our study demonstrated that $41 \%$ of patients with RA under rheumatologic care were treated with DMARD within 6 months from symptom onset. The median time to DMARD treatment was 8.4 months. The majority of patients initiated DMARD treatment at first presentation to rheumatology. The antirheumatic agents utilized at initiation of DMARD for this cohort were previously reported ${ }^{26}$. A large minority $(39.8 \%)$ started DMARD treatment in advance of receiving a definitive diagnosis. The majority of the time to DMARD treatment $(78.1 \%)$ occurred before referral to rheumatology. The most prominent predictor of increased time to treatment was concomitant musculoskeletal condition. The effects of

Personal non-commercial use only. The Journal of Rheumatology Copyright (c) 2012. All rights reserved. 
Table 2. Bivariate and multivariable-adjusted baseline* determinants of early DMARD treatment.

\begin{tabular}{|c|c|c|c|c|}
\hline \multirow[b]{2}{*}{ Variable } & \multicolumn{2}{|c|}{ Bivariate } & \multicolumn{2}{|c|}{ Multivariable $\mathrm{p}<0.50$ Stepwise Selection Model } \\
\hline & $\begin{array}{l}\text { Effect Size }^{\dagger}, \\
\quad \mathrm{n}=339\end{array}$ & $\mathrm{p}^{\dagger \dagger}$ & $\begin{array}{c}\mathrm{OR}_{\mathrm{adj}}(95 \% \mathrm{CI}) \\
\mathrm{n}=339\end{array}$ & $\begin{array}{c}\% \text { of Bootstrap } \\
\text { Models } * *, n=200\end{array}$ \\
\hline Age at symptom onset, yrs & 0.59 & 0.77 & $1.01(0.99,1.03)$ & 63.0 \\
\hline Swollen joint count & 2 & 0.05 & $1.02(0.99,1.05)$ & 59.5 \\
\hline Tender joint count & 2 & 0.05 & & 50.0 \\
\hline $\mathrm{ESR}, \mathrm{mm} / \mathrm{h}$ & 0.50 & 0.92 & & 55.5 \\
\hline $\mathrm{CRP}, \mathrm{mg} / \mathrm{l}$ & 1.00 & 0.42 & $1.00(0.99,1.00)$ & 66.0 \\
\hline Rheumatoid factor-positive & 1.00 & 1.00 & & 56.5 \\
\hline Male & 0.55 & 0.02 & $1.63(0.96,2.76)$ & 84.5 \\
\hline $\begin{array}{l}\text { Absence of concomitant MSK } \\
\text { condition }\end{array}$ & 1.59 & 0.04 & $1.87(1.12,3.11)$ & 96.0 \\
\hline $\begin{array}{l}\text { Absence of radiographic } \\
\text { evidence of erosions }\end{array}$ & 1.68 & 0.10 & $1.75(0.92,3.36)$ & 81.5 \\
\hline $\begin{array}{l}\text { Previously consulted another } \\
\text { rheumatologist }\end{array}$ & 1.60 & 0.04 & $1.86(1.15,3.01)$ & 98.5 \\
\hline Use of NSAID prior to diagnosis & s $\quad 0.90$ & 0.74 & & 49.0 \\
\hline Academic study investigator & 1.73 & 0.02 & $1.60(0.99,2.59)$ & 87.5 \\
\hline c-index $\pm \mathrm{SD}$ & NA & NA & 0.65 (NA) & $0.69 \pm 0.03$ \\
\hline
\end{tabular}

* Maximum value recorded in the clinical chart up to the date of diagnosis. Early DMARD treatment defined as treatment onset within 6 months from symptom onset. ${ }^{\dagger}$ Median difference for ordinal variables with skewed data distributions, $<6$ months to $\geq 6$ months; OR for binary variables, $<6$ months vs $\geq 6$ months. ${ }^{\dagger \dagger} \mathrm{Z}$ approximation of Wilcoxon rank-sum test for ordinal variables with skewed data distributions; Cochran Mantel-Haenszel chi-square test for $2 \times 2$ tables for binary data, positive vs negative variable. ** Percentage of 200 bootstrap $\mathrm{p}<0.5$ stepwise selection models that selected the variables. CRP: C-reactive protein; DMARD: disease-modifying antirheumatic drug; ESR: erythrocyte sedimentation rate; MSK: musculoskeletal; NA: not applicable; NSAID: nonsteroidal antiinflammatory drug; $\mathrm{OR}_{\mathrm{adj}}$; odds ratio adjusted for variables included in model.

Table 3. Bivariate and multivariable-adjusted baseline* determinants of time to DMARD treatment.

\begin{tabular}{|c|c|c|c|c|}
\hline \multirow[b]{2}{*}{ Variable } & \multicolumn{2}{|c|}{ Bivariate } & \multicolumn{2}{|c|}{$\begin{array}{c}\text { Multivariable } \mathrm{p}<0.50 \\
\text { Stepwise Selection Model }\end{array}$} \\
\hline & $\begin{array}{l}\text { Effect Size }^{\dagger} \\
\quad \mathrm{n}=339\end{array}$ & $\mathrm{p}^{\dagger \dagger}$ & $\begin{array}{l}\text { Hazard Ratio** } \\
\mathrm{n}=339\end{array}$ & $\mathrm{p}$ \\
\hline Age at symptom onset, yrs & -0.09 & 0.11 & 1.01 & 0.02 \\
\hline Swollen joint count & -0.12 & 0.03 & & \\
\hline Tender joint count & -0.11 & 0.05 & & \\
\hline $\mathrm{ESR}, \mathrm{mm} / \mathrm{h}$ & -0.08 & 0.15 & 1.01 & 0.02 \\
\hline $\mathrm{CRP}, \mathrm{mg} / \mathrm{l}$ & -0.10 & 0.08 & 1.00 & 0.48 \\
\hline Rheumatoid factor-positive & +2 & 0.72 & 1.14 & 0.33 \\
\hline Male & -96 & 0.03 & & \\
\hline Absence of concomitant MSK condition & -94 & 0.05 & 1.45 & $<0.01 * * *$ \\
\hline $\begin{array}{l}\text { Absence of radiographic evidence } \\
\text { of erosions }\end{array}$ & -160 & 0.02 & 1.28 & 0.11 \\
\hline $\begin{array}{l}\text { Previously consulted another } \\
\text { rheumatologist }\end{array}$ & -62 & 0.05 & & \\
\hline Use of NSAID prior to diagnosis & +35 & 0.59 & & \\
\hline Seen by an academic study investigator & -95 & 0.04 & 1.25 & 0.06 \\
\hline
\end{tabular}

* Maximum value recorded in the clinical chart up to the date of diagnosis. Time to DMARD treatment measured relative to date of symptom onset. ${ }^{\dagger}$ For ordinal variables, Spearman's rank correlation coefficient for variable vs days from symptom onset to DMARD treatment; for binary variables median difference in days from symptom onset to DMARD treatment for positive minus negative variable. ${ }^{\dagger \dagger}$ Spearman's rank correlation coefficient $\mathrm{p}$ value for ordinal variables; Wilcoxon rank-sum test for binary variables. ** For DMARD treatment. $* * * \mathrm{p}=0.002$. CRP: C-reactive protein: DMARD: disease-modifying antirheumatic drug; ESR: erythrocyte sedimentation rate; MSK: musculoskeletal; NSAID: nonsteroidal antiinflammatory drug. 
female sex, younger age, consulting a single versus multiple rheumatologists, consulting a nonacademic study investigator, and radiographic evidence of erosive joint damage were inconsistent across the models developed. Generally, effect sizes were small, with $\mathrm{OR}<1.9$.

Time to DMARD treatment. In this cohort, the timing of initiation of DMARD met guideline benchmarks that target the initiation of treatment within 3 months from "established" disease ${ }^{1,2,3}$. Here, established disease was interpreted as synonymous with the study definition of a definitive diagnosis. The majority of patients were treated with DMARD at first presentation to rheumatology and before documentation of a definitive diagnosis. Relative to symptom onset, the results were less encouraging: $41 \%$ were treated with DMARD within 6 months from symptom onset.

Components of time to DMARD treatment. The majority of time to DMARD treatment occurred before rheumatology referral $(78.1 \%)$. Using the start of NSAID treatment as a surrogate for a first primary care physician (PCP) visit, $51.1 \%$ of the time to DMARD treatment occurred while under the care of a PCP. NSAID are commonly prescribed by $\mathrm{PCP}$ for $\mathrm{RA}^{32}$.

The median time to patient referral to rheumatology coincided with the median time to reporting a probable diagnosis of RA. In the absence of good diagnostic tests for early disease, PCP appear to be reluctant to refer patients until they can assign a probable diagnosis ${ }^{33}$. The delay to assigning a probable diagnosis may be the consequence of a large proportion of patients with RA presenting with insidious symptoms. Data from the Norfolk Arthritis Register (NoAR) support this hypothesis ${ }^{34}$. In NoAR, estimates of RA incidence rose by $45 \%$ for women and $36 \%$ for men when the duration of symptom onset eligibility criterion increased from 1 to 5 years. The NoAR data indicate that many patients present with undifferentiated inflammatory arthritis and remain undiagnosed well beyond 6 months from symptom onset. Despite initiatives to improve the musculoskeletal examination competency of $\mathrm{PCP}^{35,36}$ and early RA referral ${ }^{37,38}$, the heterogeneity of RA near symptom onset may be a major obstacle to PCP referral to rheumatology and early DMARD treatment. Further, there is abundant data to suggest that patient preferences and psychosocial variables, and patient- and physician-physician interrelationship issues, may contribute to delays in using otherwise accessible healthcare ${ }^{10,33,39,40,41}$. In the absence of improved early disease detection methods, earlier referral to rheumatology may increase the false-positive rate of RA referrals, consume specialty resources, and adversely affect stage 2 diagnostic certainty ${ }^{42}$. Screening tools such as the self-administered early inflammatory arthritis detection tool ${ }^{43}$ hold promise in this regard.

Predictors of delays to DMARD treatment. The most prominent predictor of increased time to DMARD treatment was the existence of a concomitant musculoskeletal condition.
The foremost explanation for this association is the challenge of discriminating developing RA from established noninflammatory conditions $1,2,13,36,38$. It is noteworthy, however, that the association in this study was seen with osteoporosis independently, a disease with symptoms distinct from RA. Concomitant musculoskeletal conditions affected $40.4 \%$ of patients and their potential influence on early intervention warrants further investigation. Research to better define and validate RA-specific symptoms may be required to lessen time to treatment.

The significance of other variables we considered were less consistent across the models developed. In the logistic regression model and bootstrap validation, previously consulting another rheumatologist was associated with treatment within 6 months from symptom onset. The association may be the effect of early treatment as opposed to a cause. Because the majority of patients started DMARD treatment at their first rheumatology presentation, patients treated early with DMARD may subsequently consult additional rheumatologists to obtain a second opinion. Of the patients who consulted multiple rheumatologists, $87.4 \%$ started DMARD treatment before their first visit with the study rheumatologist, a median difference of 127 days (IQR 26-432). It is conceivable that a proportion of patients consulting multiple rheumatologists consulted the initial rheumatologist in an emergent hospital setting; however, the IQR for initial DMARD treatment suggests that this explanation may account for only a small proportion of patients consulting multiple rheumatologists. Given that $41.9 \%$ of patients with RA consult multiple rheumatologists in the setting of limited rheumatology resources, this finding warrants further investigation.

Increased time to DMARD treatment for women was suggested from the bootstrap validation data and is consistent with literature reports ${ }^{20,44,45}$. In our study, no sex-based difference existed in time from symptom onset to the initiation of NSAID; however, times to rheumatology referral and rheumatology visit were prolonged for women. These post hoc, bivariate comparisons were hypothesis-generating in character. They warrant further investigation given that female sex is predictive of disease persistence and poor outcome ${ }^{46}$.

Similarly, bootstrap validation data suggested earlier DMARD treatment among patients who consulted an academic study investigator. A higher percentage of nonacademic rheumatologists serve large geographical areas, including rural communities, where disparities in DMARD use have been linked with lack of access to specialty care ${ }^{47}$. As such, regional disparities in access to specialty care may partially account for delays prior to rheumatology referral. Nonetheless, it is noteworthy that among patients who consulted a single rheumatologist, the time from first rheumatology visit to treatment was shorter for those consulting an academic [0 days (IQR 0-30)] versus a nonacademic investigator [21 days (IQR 0-98; $\mathrm{p}=0.002$ )].

Personal non-commercial use only. The Journal of Rheumatology Copyright (c) 2012. All rights reserved. 
Similarly, care is required in the interpretation of the radiographic evidence of erosions data. At baseline, only $16.2 \%$ had radiographic evidence of erosions. Of these patients, $74.5 \%$ had first evidence of erosions documented on or before the date of initiating DMARD. Overall, however, $38.6 \%$ of patients $(n=131)$ had evidence of erosions ever reported over study followup. Of these, the majority (67.2\%) were treated with DMARD prior to the first documented evidence of erosions. For most patients, DMARD treatment was chosen prior to documented evidence of erosions. These data suggest that physicians do not rely on radiographic evidence for initial decision making about DMARD treatment.

RF positivity did not influence the timing of initiating DMARD. Others have found that positive RF triggers referral to rheumatology 20,23 . No association between RF and time to DMARD treatment was observed in the current study.

The association between older age and lesser time to DMARD treatment in the Cox model corroborates an earlier report ${ }^{20}$. Elderly onset RA may be associated with acute symptom onset ${ }^{48,49}$, and tends to have higher levels of acute-phase reactants ${ }^{48,49}$ and possibly increased erosive damage ${ }^{50}$. Older age is also associated with functional decline earlier in the disease course ${ }^{48,49}$. Although age varies with these predisposing variables, data from the Cox model in our study suggested that increasing age was an independent predictor of earlier treatment with DMARD.

Despite the known limitations with chart audits ${ }^{51}$, this may be the most appropriate study design for quality assurance-related research questions ${ }^{52}$. Our specific work was limited to patients with RA who presented to a rheumatologist. Although administrative datasets suggest that patients with RA who are under rheumatologic care may represent as little as $50 \%$ of true incident $\mathrm{RA}^{32,53}$, its diagnostic algorithms have limited accuracy ${ }^{54}$. These data suggest that a small proportion of RA patients start DMARD therapy in primary care $^{32}$. Further, time to DMARD treatment in our study was within the range reported by others ${ }^{18,19,20,21,22,23,24}$.

The number of rheumatologists who participated in this study was limited. Despite involving only 18 rheumatologists, $41.9 \%$ of included patients had previously consulted another rheumatologist, and $87.4 \%$ of them were started on DMARD therapy by that other rheumatologist. Thus, these data represent initial DMARD care from a broader sample of rheumatologists than those who specifically participated in the study. Further, on the basis of demographics, educational variables, and academic appointments, participating rheumatologists were similar to all Canadian rheumatologists $^{25}$. By randomly sampling patients at each site, selection bias toward "best cases" was avoided.

The diagnostic inception window for this cohort was June 2001 to May 2003. Over this interval, time from symp- tom onset to DMARD treatment decreased slightly ( $\mathrm{r}=$ $-0.14, \mathrm{p}=0.02)$. As reported ${ }^{26}$, temporal differences in DMARD prescription behavior also existed in this cohort. These findings support a contemporary reevaluation of this work.

Regarding the observed association between concomitant musculoskeletal disease and delays to initiation of DMARD, confounding by indication cannot be dismissed as an alternative explanation. Despite using the rheumatologists' reports of symptom onset, the low specificity of symptoms may bias the results toward longer durations for patients with concomitant musculoskeletal conditions, such as osteoarthritis or fibromyalgia, but surely not osteoporosis. Other literature suggests that patient preferences, psychosocial factors, and patient-physician and physician-physician interrelationship issues hamper rheumatology referral ${ }^{10,33,39,40,41}$. The chart-audit design was not conducive to collection of these potentially predictive variables. Of regional consequence, the literature suggests that geographical displacement may also influence access to care ${ }^{53}$. Our study did not specifically consider this issue, which may be predictive of time to DMARD treatment.

In this national, incident cohort of randomly selected, clinically diagnosed patients with RA, $41 \%$ started DMARD treatment within 6 months from symptom onset. The average patient was treated at first presentation to rheumatology, prior to the documentation of a definitive diagnosis. The majority of time to DMARD treatment occurred before referral to rheumatology. A concomitant musculoskeletal condition was predictive of increased time to DMARD treatment. Earlier referral to rheumatology and distinction of RA from other musculoskeletal conditions may allow for decreased time to treatment. A contemporary reevaluation is required to corroborate and further investigate the findings from this study.

\section{ACKNOWLEDGMENT}

The authors thank Dr. Ahmed Bayoumi and Dr. Paul Fortin for their respective reviews of this University of Toronto Institute of Medical Science Master of Science thesis project. Data extraction support from Diane Ferland, Donna McBain, and Melanie Hargrove-Boucher, and editing support from Annette Wilkins is gratefully acknowledged.

\section{REFERENCES}

1. American College of Rheumatology Subcommittee on Rheumatoid Arthritis Guidelines. Guidelines for the management of rheumatoid arthritis: 2002 update. Arthritis Rheum 2002;46:328-46.

2. Combe B, Landewe R, Lukas C, Bolosiu HD, Breedveld F, Dougados M, et al. EULAR recommendations for the management of early arthritis: Report of a task force of the European Standing Committee for International Clinical Studies Including Therapeutics (ESCISIT). Ann Rheum Dis 2007;66:34-45.

3. Smolen JS, Landewe R, Breedveld FC, Dougados M, Emery P, Gaujoux-Viala C, et al. EULAR recommendations for the management of rheumatoid arthritis with synthetic and biological disease modifying antirheumatic drugs. Ann Rheum Dis 2010;69:964-75. Personal non-commercial use only. The Journal of Rheumatology Copyright @ (2012. All rights reserved. 
4. Boers M, Verhoeven AC, Markusse HM, van de Laar MA, Westhovens R, van Denderen JC, et al. Randomised comparison of combined step-down prednisolone, methotrexate and sulphasalazine with sulphasalazine alone in early rheumatoid arthritis. Lancet 1997;350:309-18.

5. van der Heide A, Jacobs JW, Bijlsma JW, Heurkens AH, van Booma-Frankfort C, van der Veen MJ, et al. The effectiveness of early treatment with "second-line" antirheumatic drugs. A randomized, controlled trial. Ann Intern Med 1996;124:699-707.

6. Egsmose C, Lund B, Borg G, Pettersson H, Berg E, Brodin U, et al. Patients with rheumatoid arthritis benefit from early 2 nd line therapy: 5 year followup of a prospective double blind placebo controlled study. J Rheumatol 1995;22:2208-13.

7. HERA Study Group. A randomized trial of hydroxychloroquine in early rheumatoid arthritis: The HERA Study. Am J Med 1995;98:156-68.

8. Mottonen T, Hannonen P, Korpela M, Nissila M, Kautiainen H, Ilonen J, et al. Delay to institution of therapy and induction of remission using single-drug or combination-disease-modifying antirheumatic drug therapy in early rheumatoid arthritis. Arthritis Rheum 2002;46:894-8.

9. Green M, Marzo-Ortega H, McGonagle D, Wakefield R, Proudman $\mathrm{S}$, Conaghan $\mathrm{P}$, et al. Persistence of mild, early inflammatory arthritis: The importance of disease duration, rheumatoid factor, and the shared epitope. Arthritis Rheum 1999;42:2184-8.

10. Quinn MA, Green MJ, Marzo-Ortega H, Proudman S, Karim Z, Wakefield RJ, et al. Prognostic factors in a large cohort of patients with early undifferentiated inflammatory arthritis after application of a structured management protocol. Arthritis Rheum 2003;48:3039-45.

11. Wiles NJ, Lunt M, Barrett EM, Bukhari M, Silman AJ, Symmons DP, et al. Reduced disability at five years with early treatment of inflammatory polyarthritis: Results from a large observational cohort, using propensity models to adjust for disease severity. Arthritis Rheum 2001;44:1033-42.

12. Aletaha D, Smolen J, Ward MM. Measuring function in rheumatoid arthritis: Identifying reversible and irreversible components. Arthritis Rheum 2006;54:2784-92.

13. Quinn MA, Green MJ, Conaghan P, Emery P. How do you diagnose rheumatoid arthritis early? Best Pract Res Clin Rheumatol 2001;15:49-66

14. Arnett FC, Edworthy SM, Bloch DA, McShane DJ, Fries JF, Cooper NS, et al. The American Rheumatism Association 1987 revised criteria for the classification of rheumatoid arthritis. Arthritis Rheum 1988;31:315-24.

15. Hulsemann JL, Zeidler H. Diagnostic evaluation of classification criteria for rheumatoid arthritis and reactive arthritis in an early synovitis outpatient clinic. Ann Rheum Dis 1999;58:278-80.

16. Harrison BJ, Symmons DP, Barrett EM, Silman AJ. The performance of the 1987 ARA classification criteria for rheumatoid arthritis in a population based cohort of patients with early inflammatory polyarthritis. J Rheumatol 1998;25:2324-30.

17. Aletaha D, Neogi T, Silman AJ, Funovits J, Felson DT, Bingham CO, et al. 2010 Rheumatoid arthritis classification criteria: An American College of Rheumatology/European League Against Rheumatism Collaborative Initiative. Arthritis Rheum 2010;62:2569-81.

18. Chan KW, Felson DT, Yood RA, Walker AM. The lag time between onset of symptoms and diagnosis of rheumatoid arthritis. Arthritis Rheum 1994;37:814-20.

19. Irvine S, Munro R, Porter D. Early referral, diagnosis, and treatment of rheumatoid arthritis: Evidence for changing medical practice. Ann Rheum Dis 1999;58:510-3.

20. Hernandez-Garcia C, Vargas E, Abasolo L, Lajas C, Bellajdell B, Morado IC, et al. Lag time between onset of symptoms and access to rheumatology care and DMARD therapy in a cohort of patients with rheumatoid arthritis. J Rheumatol 2000;27:2323-8.

21. Kiely P, Williams R, Walsh D, Young A. Early Rheumatoid Arthritis Network. Contemporary patterns of care and disease activity outcome in early rheumatoid arthritis: The ERAN cohort. Rheumatology 2009;48:57-60.

22. Robinson PC, Taylor WJ. Time to treatment in rheumatoid arthritis: Factors associated with time to treatment initiation and urgent triage assessment of general practitioner referrals. J Clin Rheumatol 2010;16:267-73.

23. Sokka T, Kautiainen H, Toloza S, Mäkinen H, Verstappen SMM, Hetland ML, et al. QUEST-RA: quantitative clinical assessment of patients with rheumatoid arthritis seen in standard rheumatology care in 15 countries. Ann Rheum Dis 2007;66:1491-6.

24. Jamal S, Alibhai SM, Badley EM, Bombardier C. Time to treatment for new patients with rheumatoid arthritis in a major metropolitan city. J Rheumatol 2011;38:1282-8.

25. Canadian Medical Association. Canadian medical directory. Toronto: Business Information Group, a division of HCN Publications Company; 2006.

26. Tavares R, Pope JE, Tremblay JL, Thorne C, Bykerk VP, Lazovskis $\mathrm{J}$, et al. Early management of newly diagnosed rheumatoid arthritis by Canadian rheumatologists: A national, multicenter, retrospective cohort. J Rheumatol 2011;38:2342-5.

27. Steyerberg EW, Eijkemans MJ, Harrell FE Jr, Habbema JD. Prognostic modeling with logistic regression analysis: In search of a sensible strategy in small data sets. Med Decis Making 2001;21:45-56.

28. Schafer JL. Analysis of incomplete multivariate data. New York: Chapman \& Hall; 1997.

29. Altman DG. Practical statistics for medical research. London: Chapman \& Hall; 1991.

30. Smith T, Smith B, Ryan MAK. Survival analysis using Cox proportional hazards modeling for single and multiple event time data. Proceedings of the Twenty-Eighth Annual SAS Users Group International Conference. Cary, NC: SAS Institute Inc.; 2003.

31. Efron B. An introduction to the bootstrap. New York: Chapman \& Hall; 1993.

32. Lacaille D, Anis AH, Guh DP, Esdaile JM. Gaps in care for rheumatoid arthritis: A population study. Arthritis Rheum 2005;53:241-8

33. Suter LG, Fraenkel L, Holmboe ES. What factors account for referral delays for patients with suspected rheumatoid arthritis? Arthritis Rheum 2006;55:300-5.

34. Wiles N, Symmons DP, Harrison B, Barrett E, Barrett JH, Scott DG, et al. Estimating the incidence of rheumatoid arthritis: Trying to hit a moving target? Arthritis Rheum 1999;42:1339-46.

35. Petrella RJ, Davis P. Improving management of musculoskeletal disorders in primary care: The Joint Adventures Program. Clin Rheumatol 2007;26:1061-6.

36. Glazier RH, Badley EM, Lineker SC, Wilkins AL, Bell MJ. Getting a grip on arthritis: An educational intervention for the diagnosis and treatment of arthritis in primary care. J Rheumatol 2005;32:137-42.

37. Emery P, Breedveld FC, Dougados M, Kalden JR, Schiff MH, Smolen JS. Early referral recommendation for newly diagnosed rheumatoid arthritis: Evidence based development of a clinical guide. Ann Rheum Dis 2002;61:290-7.

38. Dore RK, Clements PJ, Fox RI, Furst DE, Kaplan H, Kitridou RC. Guidelines for rheumatology referral. Atlanta: American College of Rheumatology; 1996.

39. Glazier RH, Dalby DM, Badley EM, Hawker GA, Bell MJ, Buchbinder R, et al. Management of the early and late presentations of rheumatoid arthritis: A survey of Ontario primary care physicians. CMAJ 1996;155:67.

40. Gruppen LD, Wolf FM, Van Voorhees C, Stross JK. The influence

Personal non-commercial use only. The Journal of Rheumatology Copyright (C) 2012. All rights reserved. 
of general and case-related experience on primary care treatment decision making. Arch Intern Med 1988;148:2657-63.

41. Gamez-Nava JI, Gonzalez-Lopez L, Davis P, Suarez-Almazor ME Referral and diagnosis of common rheumatic diseases by primary care physicians. Br J Rheumatol 1998;37:1215-9.

42. Guillemin F, Saraux A, Fardellone P, Guggenbuhl P, Behier JM, Coste J, et al. Detection of cases of inflammatory rheumatic disorders: Performance of a telephone questionnaire designed for use by patient interviewers. Ann Rheum Dis 2003;62:957-63.

43. Bell MJ, Tavares R, Guillemin F, Bykerk VP, Tugwell P, Wells GA. Development of a self-administered early inflammatory arthritis detection tool. BMC Musculoskelet Disord 2010;11:50.

44. Lard LR, Huizinga TW, Hazes JM, Vlieland TP. Delayed referral of female patients with rheumatoid arthritis. J Rheumatol 2001;28:2190-2.

45. Palm O, Purinszky E. Women with early rheumatoid arthritis are referred later than men. Ann Rheum Dis 2005;64:1227-8.

46. Harrison B, Symmons D. Early inflammatory polyarthritis: Results from the Norfolk Arthritis Register with a review of the literature. II. Outcome at three years. Rheumatology 2000;39:939-49.

47. Shipton D, Glazier RH, Guan J, Badley EM. Effects of use of specialty services on disease-modifying antirheumatic drug use in the treatment of rheumatoid arthritis in an insured elderly population. Med Care 2004;42:907-13.
48. Olivieri I, Palazzi C, Peruz G, Padula A. Management issues with elderly-onset rheumatoid arthritis: An update. Drugs Aging 2005;22:809-22.

49. Papadopoulos IA, Katsimbri P, Alamanos Y, Voulgari PV, Drosos AA. Early rheumatoid arthritis patients: Relationship of age. Rheumatol Int 2003;23:70-4

50. Peltomaa R, Leirisalo-Repo M, Helve T, Paimela L. Effect of age on 3 year outcome in early rheumatoid arthritis. J Rheumatol 2000;27:638-43.

51. Pan L, Fergusson D, Schweitzer I, Hebert PC. Ensuring high accuracy of data abstracted from patient charts: The use of a standardized medical record as a training tool. J Clin Epidemiol 2005;58:918-23.

52. Worster A, Haines T. Advanced statistics: Understanding medical record review (MRR) studies. Acad Emerg Med 2004;11:187-92.

53. Badley EM. Arthritis in Canada: What do we know and what should we know? J Rheumatol Suppl. 2005 Jan;72:39-41.

54. Kim SY, Servi A, Polinski JM, Mogun H, Weinblatt ME, Katz JN, et al. Validation of rheumatoid arthritis diagnoses in health care utilization data. Arthritis Res Ther 2011;13:R32. 